\title{
Diagnostic Value of Endoscopic Ultrasonography for Common Bile Duct Dilatation without Identifiable Etiology Detected from Cross- Sectional Imaging
}

\author{
Nonthalee Pausawasdi ${ }^{1,2}$, Penprapai Hongsrisuwan', Lubna Kamani ${ }^{1,3}$, Kotchakon Maipang ${ }^{1,2}$, and Phunchai Charatcharoenwitthaya ${ }^{1,2}$ \\ ${ }^{1}$ Siriraj GI Endoscopy Center, Faculty of Medicine Siriraj Hospital, Mahidol University, Bangkok, ${ }^{2}$ Division of Gastroenterology, \\ Department of Medicine, Faculty of Medicine Siriraj Hospital, Mahidol University, Bangkok, Thailand, ${ }^{3}$ Liaquat National Hospital and \\ Aga Khan University Hospital, Karachi, Pakistan
}

Background/Aims: Endoscopic ultrasonography (EUS) is warranted when cross-sectional imaging demonstrates common bile duct (CBD) dilatation without identifiable causes. This study aimed to assess the diagnostic performance of EUS in CBD dilatation of unknown etiology.

Methods: Retrospective review of patients with dilated CBD without definite causes undergoing EUS between 2012 and 2017.

Results: A total of 131 patients were recruited. The mean age was $63.2 \pm 14.1$ years. The most common manifestation was abnormal liver chemistry $(85.5 \%)$. The mean CBD diameter was $12.2 \pm 4.1 \mathrm{~mm}$. The area under the receiver operating characteristic curve (AUROC) of EUS-identified pathologies, including malignancy, choledocholithiasis, and benign biliary stricture (BBS), was 0.98 (95\% confidence interval [CI], 0.95-1.00). The AUROC of EUS for detecting malignancy, choledocholithiasis, and BBS was 0.91 (95\% CI, 0.85-0.97), 1.00 (95\% CI, 1.00-1.00), and 0.93 (95\% CI, 0.87-0.99), respectively. Male sex, alanine aminotransferase $\geq 3 \times$ the upper limit of normal (ULN), alkaline phosphatase $\geq 3 \times$ the ULN, and intrahepatic duct dilatation were predictors for pathological obstruction, with odds ratios of 5.46 (95\%CI, 1.74-17.1), 5.02 (95\% CI, 1.48-17.0), 4.63 (95\% CI, 1.1-19.6), and 4.03 (95\% CI, 1.3711.8), respectively.

Conclusions: EUS provides excellent diagnostic value in identifying the etiology of CBD dilatation detected by cross-sectional imaging. Clin Endosc 2022;55:122-127

Key Words: Common bile duct; Diagnostic imaging; Endosonography; Three-dimensional imaging

\section{INTRODUCTION}

Cross-sectional imaging, including multi-detector computed tomography (MDCT), magnetic resonance imaging (MRI), and magnetic resonance cholangiopancreatography (MRCP), have been widely used to evaluate the pancreaticobiliary sys-

\footnotetext{
Received: April 6, 2021 Revised: June 14, 2021

Accepted: July 7, 2021

Correspondence: Nonthalee Pausawasdi

Division of Gastroenterology, Department of Medicine, Siriraj Hospital, WangLang Road, Bangkoknoi, Bangkok 10700, Thailand

Tel: +662 419-7282, Fax: +662 411-5013, E-mail: nonthaleep7@gmail.com

ORCID: https://orcid.org/0000-0002-3737-8555

(c) This is an Open Access article distributed under the terms of the Creative Commons Attribution Non-Commercial License (http://creativecommons.org/ licenses/by-nc/3.0) which permits unrestricted non-commercial use, distribution, and reproduction in any medium, provided the original work is properly cited.
}

tem. In detecting pancreatic and biliary disease, MDCT and MRI have a sensitivity of $70 \%-95 \%$ and $85 \%-96 \%$ and a specificity of $75 \%-85 \%$ and $89 \%-98 \%$, respectively. ${ }^{1-5}$ Despite their excellent diagnostic performance, the evaluation of the distal bile duct or ampullary area is often limited. In asymptomatic patients, dilated common bile ducts (CBDs) on imaging may be influenced by age, sex, body mass index, and cholecystectomy history. The common pathological causes of CBDs dilatation are choledocholithiasis, periampullary carcinoma, and benign biliary stricture (BBS); however, these lesions can be missed by MDCT, MRI, and MRCP. ${ }^{6-12}$ Endoscopic retrograde cholangiopancreatography (ERCP) has historically been one of the most accurate diagnostic procedures for pancreatic and biliary diseases. However, it should only be done for therapeutic purposes due to its invasive nature and potential lethal complications, such as post-ERCP pancreatitis, bleeding, and 
perforation. Endoscopic ultrasonography (EUS) has evolved as a tool for evaluating hepatobiliary and pancreatic diseases. There is a dearth of literature regarding the utility of EUS in outlining a dilated CBDs. ${ }^{13}$ This study aimed to evaluate the diagnostic yield of EUS in dilated CBDs without identifiable causes on MDCT or MRI with or without MRCP findings.

\section{MATERIALS AND METHODS}

\section{Study population}

The 2012-2017 EUS database at a tertiary care center was retrospectively reviewed. The study protocol was approved by the institutional review board and was adapted to the ethical guidelines of the Declaration of Helsinki. Patients who underwent EUS due to CBDs dilatation without definite etiology detected by MDCT or MRI with or without MRCP were identified. The inclusion criteria were as follows: 1) CBDs dilatation, defined as CBDs diameter $\geq 7 \mathrm{~mm}$ in patients with gall bladder in situ or $\geq 10 \mathrm{~mm}$ in post-cholecystectomy patients and 2) no causes of CBDs dilatation identified by MDCT or MRI with or without MRCP. The exclusion criteria were as follows: 1) patients with definite causes of CBDs dilatation identified by cross-sectional imaging and 2) patients without available MDCT, MRI, or MRCP for review.

\section{Clinical, laboratory, and radiological data}

All EUS procedures were performed with either a radial (GF-UE160-AL5; Olympus, Tokyo, Japan) or linear (GFUC140P-AL5; Olympus, Tokyo, Japan) echoendoscope by an experienced endoscopist who had performed more than 2,000 EUS procedures. Demographic data, clinical presentations, laboratory results, radiological findings, EUS findings, cytopathological results, and follow-up data of all included patients were collected. The definite diagnosis was determined by the results of ERCP, cytology, or histology obtained from EUS-guided tissue acquisition (EUS-TA), surgical pathology, and clinical, laboratory, and radiological follow-up for at least 12 months.

\section{Definition}

Choledocholithiasis was determined by visualization of the stones in the CBDs during ERCP. Malignancy was confirmed by cytology or histology obtained by EUS-TA, or surgical pathology. If the tissue diagnosis could not be obtained, clinical, laboratory, and radiological follow-up was required for at least 12 months. BBS was defined as narrowing of the distal CBDs diameter without visualization of stones or masses and negative cytology or histology obtained by EUS-TA or ERCP, combined with no progression of bile duct dilatation and interval symptoms during a 12-month follow-up of clinical condition, laboratory, and radiological studies. If surgical pathology was available, BBS was defined as the absence of malignancy. CBDs dilatation without pathological causes was determined by the absence of progression of bile duct dilatation and interval symptoms during a 12-month follow-up of clinical condition, laboratory, and radiological studies.

\section{Statistical analysis}

Descriptive statistics were computed for demographic, clinical, and laboratory data. For normally distributed quantitative variables, results are expressed as means and standard deviations; otherwise, medians and ranges are reported. Qualitative variables were summarized as counts and percentages. The area under the receiver operating characteristic curve (AUROC) was calculated to evaluate the overall accuracy of EUS for identifying the causes of bile duct dilatation. The predictive ability was further analyzed by calculating the sensitivity, specificity, positive likelihood ratio, negative likelihood ratio, positive predictive value (PPV), and negative predictive value (NPV) with their $95 \%$ confidence interval (CI)s. Logistic regression models were used to evaluate the relationship between baseline characteristics and the presence or absence of pathology. All statistical tests were performed at the conventional two-tailed $\alpha$-level of 0.05 . SPSS Statistics (version 18.0; SPSS Inc., Chicago, IL, USA) was used for all analyses.

\section{RESULTS}

\section{Characteristics of the study cohort}

The EUS database showed that 2,954 patients underwent diagnostic EUS during the study period. A total of $175 \mathrm{pa}-$ tients underwent EUS for CBDs dilatation without identifiable causes. Forty-four patients were excluded because of unavailable radiological studies for review. Among the 131 included patients, the mean age was $63.2 \pm 14.1$ years, $47.3 \%$ were male, and the most common clinical manifestations were abnormal liver function tests (85.5\%), jaundice (48.9\%), and abdominal pain (48.1\%). The mean CBDs diameter was $12.2 \pm 4.1 \mathrm{~mm}$, and $58 \%$ had coexisting intrahepatic duct (IHD) dilatation. Among abnormal liver function tests, elevated total bilirubin (median, $2.3 \mathrm{mg} / \mathrm{dL}$; range, 0.2-37.8), aspartate aminotransferase (median, $66 \mathrm{IU} / \mathrm{L}$ ), alanine aminotransferase (ALT) (median, $66 \mathrm{IU} / \mathrm{L}$ ), and alkaline phosphatase (ALP) (median, $249 \mathrm{IU} / \mathrm{L}$ ) were detected (Table 1).

\section{Definite etiology of CBD dilatation}

EUS detected the causes of CBDs dilatation in 88 of 131 patients (67\%). Among the 131 patients, 41 patients (31\%) had 
Table 1. Baseline Characteristics of the Study Population

\begin{tabular}{lc}
\hline Baseline Characteristics & Values \\
\hline Number & 131 \\
Male gender & $62(47.3)$ \\
Age (years) & $63.2 \pm 14.1$ \\
Body mass index $\left(\mathrm{kg} / \mathrm{m}^{2}\right)$ & $21.8 \pm 3.5$ \\
History of cholecystectomy & $19(14.5)$ \\
Clinical presentation & \\
Abnormal liver function test & $112(85.5)$ \\
Jaundice & $64(48.9)$ \\
Abdominal pain & $63(48.1)$ \\
Fever & $19(14.5)$ \\
Constitutional symptoms & $19(14.5)$ \\
Weight loss & $29(22.1)$ \\
Palpable gallbladder & $5(3.8)$ \\
Laboratory finding & \\
AST (IU/L) & $66(7-611)$ \\
ALT (IU/L) & $66(7-611)$ \\
ALP (IU/L) & $249(28-1,630)$ \\
Gamma glutamyl transpeptidase (IU/L) & $445(35-1,906)$ \\
Total bilirubin (mg/dL) & $2.3(0.2-37.8)$ \\
CA 19-9 (IU/L) & $62.9(20-52,843)$ \\
Imaging findings & \\
Common bile duct diameter (mm) & $12.2 \pm 4.1$ \\
Intrahepatic duct dilatation & $77(58.8)$ \\
\hline Chronic pancreatitis & $14(10.7)$ \\
\hline
\end{tabular}

Data are presented as the number (\%) or mean \pm standard deviation.

ALP, alkaline phosphatase; ALT, alanine aminotransferase; AST, aspartate aminotransferase; CA, carbohydrate antigen. malignancy, consisting of distal cholangiocarcinoma (51.2\%), ampullary adenocarcinoma (24.3\%), pancreatic adenocarcinoma $19.5 \%)$, gallbladder carcinoma (2.5\%), and duodenal adenocarcinoma (2.5\%). A total of 24 (18.3\%), 23 (17.6\%), and 43 (33\%) patients had choledocholithiasis, BBS, and dilated CBDs without a pathological cause of obstruction, respectively.

\section{Diagnostic performance of EUS}

The diagnostic performance of EUS in detecting the causes of CBDs dilatation was evaluated, as shown in Table 2. EUS had an excellent diagnostic performance for identifying the etiology of CBDs dilatation with an AUROC, sensitivity, specificity, PPV, and NPV of 0.98 (95\% CI, 0.95-1.00), 100\% (95\% CI, 95.8-100), 95.6\% (95\% CI, 84.9-99.5), 97.7\% (95\% CI, 92.0-99.7), and 100\% (95\% CI, 91.8-100), respectively. Furthermore, we assessed the diagnostic accuracy of EUS for each diagnosis. Among all the diagnoses, EUS performed the best in detecting choledocholithiasis with an AUROC, sensitivity, and specificity of 1.00 (95\% CI, 1.00-1.00), 100\% (95\% CI, 85.8-100), and 100\% (95\% CI, 96.6-100), respectively. For malignancy, EUS was $82.9 \%$ (95\% CI, 67.9-92.8) sensitive and $98.9 \%$ (95\% CI, 94.0-100) specific with an AUROC of 0.91 (95\% CI, 0.85-0.97). For BBS, EUS had an AUROC of 0.93 (95\% CI, 0.87-0.99) with a high NPV of 98.1\% (95\% CI, 93.2-99.8).

\section{Predictors for determining the presence of pathological obstruction}

Multivariate analysis showed that male sex, ALT $\geq 3 \times$ the upper limit of normal (ULN), ALP $\geq 3 \times$ the ULN, and IHD dilatation were significant predictors for pathological obstruction, with odds ratios of 5.46 (95\% CI, 1.74-17.1), 5.02 (95\% CI, 1.48-17.0), 4.63 (95\% CI, 1.1-19.6) and 4.03 (95\% CI, $1.37-11.8$ ), respectively (Table 3).

Table 2. Diagnostic Performance of Endoscopic Ultrasonography for Identifying Causes of Common Bile Duct Dilatation

\begin{tabular}{|c|c|c|c|c|c|c|c|}
\hline Definite diagnosis & $\begin{array}{l}\text { AUROC } \\
(95 \% \mathrm{CI})\end{array}$ & Sensitivity $(95 \% \mathrm{CI})$ & Specificity $(95 \%$ CI $)$ & $\begin{array}{c}\text { LR+ } \\
(95 \% \mathrm{CI})\end{array}$ & $\begin{array}{c}\text { LR- } \\
(95 \% \text { CI })\end{array}$ & $\begin{array}{c}\text { PPV } \\
(95 \% \mathrm{CI})\end{array}$ & $\begin{array}{c}\text { NPV } \\
(95 \% \mathrm{CI})\end{array}$ \\
\hline Pathological obstruction & $\begin{array}{c}0.98 \\
(0.95-1.00)\end{array}$ & $\begin{array}{c}100 \\
(95.8-100)\end{array}$ & $\begin{array}{c}95.6 \\
(84.9-99.5)\end{array}$ & $\begin{array}{c}22.5 \\
(5.81-87.2)\end{array}$ & 0 & $\begin{array}{c}97.7 \\
(92.0-99.7)\end{array}$ & $\begin{array}{c}100 \\
(91.8-100)\end{array}$ \\
\hline Choledocholithiasis & $\begin{array}{c}1.00 \\
(1.00-1.00)\end{array}$ & $\begin{array}{c}100 \\
(85.8-100)\end{array}$ & $\begin{array}{c}100 \\
(96.6-100)\end{array}$ & - & 0 & $\begin{array}{c}100 \\
(85.8-100)\end{array}$ & $\begin{array}{c}100 \\
(96.6-100)\end{array}$ \\
\hline Malignancy & $\begin{array}{c}0.91 \\
(0.85-0.97)\end{array}$ & $\begin{array}{c}82.9 \\
(67.9-92.8)\end{array}$ & $\begin{array}{c}98.9 \\
(94.0-100)\end{array}$ & $\begin{array}{c}74.6 \\
(10.6-527)\end{array}$ & $\begin{array}{c}0.17 \\
(0.09-0.34)\end{array}$ & $\begin{array}{c}97.1 \\
(85.1-99.9)\end{array}$ & $\begin{array}{c}92.7 \\
(85.6-97.0)\end{array}$ \\
\hline Benign biliary stricture & $\begin{array}{c}0.93 \\
(0.87-0.99)\end{array}$ & $\begin{array}{c}91.7 \\
(73.0-99.0)\end{array}$ & $\begin{array}{c}94.4 \\
(88.3-97.9)\end{array}$ & $\begin{array}{c}16.5 \\
(751-36.2)\end{array}$ & $\begin{array}{c}0.09 \\
(0.02-0.33)\end{array}$ & $\begin{array}{c}78.6 \\
(59.0-91.7)\end{array}$ & $\begin{array}{c}98.1 \\
(93.2-99.8)\end{array}$ \\
\hline
\end{tabular}

AUROC, area under the receiver operating characteristic curve; CI, confidence interval; LR+, positive likelihood ratio; LR-, negative likelihood ratio; NPV, negative predictive value; PPV, positive predictive value. 
Table 3. Predictive Factors for Pathological Obstruction and No Pathological Lesion Among Patients with Common Bile Duct Dilatation

\begin{tabular}{|c|c|c|c|c|}
\hline \multirow{2}{*}{ Variables } & \multicolumn{2}{|c|}{ Univariate analysis } & \multicolumn{2}{|c|}{ Multivariate analysis } \\
\hline & OR $(95 \% \mathrm{CI})$ & $p$-value & OR $(95 \% \mathrm{CI})$ & $p$-value \\
\hline \multicolumn{5}{|l|}{ Pathological obstruction } \\
\hline Male gender & $4.10(1.79-8.97)$ & 0.001 & $5.46(1.74-17.1)$ & 0.004 \\
\hline History of cholecystectomy & $0.38(0.14-1.01)$ & 0.052 & & \\
\hline Jaundice & $20.9(6.80-64.2)$ & $<0.001$ & & \\
\hline Abdominal pain & $2.24(1.05-4.76)$ & 0.036 & & \\
\hline Fever & $10.8(1.39-83.9)$ & 0.023 & & \\
\hline Constitutional symptoms & $4.91(1.18-22.3)$ & 0.040 & & \\
\hline Total bilirubin $>5 \mathrm{mg} / \mathrm{dL}$ & $5.41(2.05-14.3)$ & 0.001 & & \\
\hline AST $>3 \times$ ULN & $2.57(1.06-6.27)$ & 0.038 & & \\
\hline ALT $>3 \times$ ULN & $4.06(1.62-10.2)$ & 0.003 & $5.02(1.48-17.0)$ & 0.009 \\
\hline $\mathrm{ALP}>3 \times \mathrm{ULN}$ & $8.17(2.33-28.7)$ & 0.001 & $4.63(1.10-19.6)$ & 0.037 \\
\hline Intrahepatic biliary dilatation & $2.83(1.33-5.99)$ & 0.007 & $4.03(1.37-11.8)$ & 0.011 \\
\hline Intraabdominal lymphadenopathy & $7.36(0.92-58.8)$ & 0.060 & & \\
\hline
\end{tabular}

ALP, alkaline phosphatase; ALT, alanine aminotransferase; AST, aspartate aminotransferase; CI, confidence interval; OR, odds ratio; ULN, upper limit of normal

\section{DISCUSSION}

CBDs dilatation without a discernible cause is not an unexpected finding on cross-sectional imaging. EUS is generally performed in cases of unexplained CBDs dilatation to evaluate the distal bile duct and ampullary area. Nonetheless, evidence-based guidelines have not been established for this clinical setting because of a lack of data. Retrospective studies have evaluated the diagnostic yield of EUS for causes of dilated CBDs, particularly in asymptomatic patients with unexplained CBDs dilatation with both normal and elevated serum liver enzymes. ${ }^{6,8,12,14,15}$ EUS was able to detect bile duct pathologies, including dilated CBDs with no obvious etiology, in cross-sectional imaging studies of $6 \%-21 \%$ of asymptomatic patients with normal liver chemistry. ${ }^{8,14,15}$ For those with combined CBDs dilatation and abnormal liver chemistry, 50\%-100\% had pathologies detected by EUS. ${ }^{12,15}$ These results emphasized the importance of EUS in this setting; nonetheless, the diagnostic accuracy is yet to be explored.

In the current study, approximately $50 \%$ of the patients were symptomatic, and the majority had abnormal liver chemistry. EUS detected bile duct pathologies in $67 \%$ of the patients with inconclusive MDCT or MRI with or without MRCP, and the diagnostic performance of EUS in detecting pathologic lesions was excellent, with an AUROC of 0.98. In contrast to other studies, the most common pathologic etiology in our study was malignant obstruction, accounting for one-third of the cohort, with distal cholangiocarcinoma being found in $51 \%$. Choledocholithiasis was the second most common etiology, accounting for $18.3 \%$, while most studies showed that choledocholithiasis was the most common cause, with rates up to nearly $40 \%$, followed by malignancy. We hypothesized that the discrepancy between our results and those of other studies could be attributed to the differences in patient characteristics, including presenting symptoms and the degree of liver chemistry abnormalities. Studies in asymptomatic patients with normal liver chemistry have demonstrated a lower percentage of abnormalities and malignancies detected by EUS. In contrast, $49 \%$ of our patients presented with jaundice and a mean total bilirubin level of $5 \mathrm{mg} / \mathrm{dL}$, suggesting underlying pathological bile duct obstruction. Furthermore, the most common malignancy was distal cholangiocarcinoma, which could be difficult to identify using a MDCT scan or MRI. EUS has increasingly become the imaging tool of choice of malignant etiology in dilated CBDs due to its high sensitivity and accuracy, especially in patients with distal biliary obstruction. ${ }^{16,17}$ Prior studies have reported a sensitivity of EUS in detecting biliary malignancy, including hilar cholangiocarcinoma, ranging from 40\%$90 \%{ }^{18-20}$ In addition, there has been a report of EUS detection of distal CBD tumor, whereas CT scan and MRCP suggested stone formation. ${ }^{21}$ The current study underscores the excellent diagnostic performance of EUS in diagnosing malignancy as a 
cause of bile duct dilatation with an AUROC of 0.91 and specificity of $98.9 \%$.

The exceptional diagnostic accuracy of EUS in detecting choledocholithiasis has been widely accepted. Although EUS and MRCP were comparable in terms of sensitivity, specificity, and accuracy for detecting choledocholithiasis, ${ }^{22}$ EUS has detected very small choledocholithiasis missed by MDCT and MRCP with 100\% diagnostic accuracy, avoiding unnecessary ERCP and surgery. ${ }^{21,23}$ Scheiman et al. ${ }^{24}$ suggested that implementing the initial EUS strategy to evaluate patients with suspected biliary disease had the greatest cost-utility, resulting in less unnecessary ERCPs and ERCP-related complications. ${ }^{24}$ Similarly, our results showed that EUS performed the best in detecting choledocholithiasis with the AUROC of 1.0. It is important to point out that EUS detection of choledocholithiasis has been reported in CBDs dilatation of unknown etiology in patients with both normal and abnormal liver chemistry, highlighting the necessity of EUS in managing this clinical scenario. Factors that may help predict pathological obstruction are male sex, serum ALT level $\geq 3 \times$ the ULN, serum ALP $\geq 3 \times$ the ULN, and IHD dilatation. Thus, when the dilated bile duct was noted on cross-sectional imaging along with the above parameters, further investigation with EUS is warranted. In contrast, clinical follow-up without further invasive investigations might be sufficient in patients without these parameters. Oppong et al. ${ }^{14}$ reported that a history of cholecystectomy, which was identified in $36 \%$ of the cases, is a causative factor for non-obstructive CBDs dilatation. In contrast, only $14 \%$ of our patients had prior cholecystectomy.

This study was limited by its retrospective nature and the need to use clinical follow-up as part of the definite diagnosis instead of undergoing ERCP or surgery in all cases. However, the strength of the study was high-quality radiologic imaging in all recruited patients and a long-term follow-up of at least 12 months.

In conclusion, EUS is a useful modality for evaluating CBDs dilatation in inconclusive MDCT, MRI, or MRCP. It should be routinely performed for clinically or biochemically indicated pancreatobiliary diseases. The excellent diagnostic performance of EUS could help avoid unnecessary ERCP or surgery in clinical practice.

Conflicts of Interest

The authors have no potential conflicts of interest.

Funding
None.

Author Contributions

Conceptualization: Nonthalee Pausawasdi, Phunchai Charatcharoenwitthaya
Data curation: Penprapai Hongsrisuwan

Formal analysis: NP, PH, PC

Investigation: $\mathrm{NP}, \mathrm{PH}$

Methodology: NP, PH

Project administration: NP

Supervision: NP

Validation: NP

Visualization: NP

Writing-original draft: PH, Lubna Kamani, Kotchakon Maipang

Writing-review and editing: NP, KM, PC

ORCID
Nonthalee Pausawasdi Penprapai Hongsrisuwan Lubna Kamani

Kotchakon Maipang

Phunchai Charatcharoenwitthaya https://orcid.org/0000-0002-3737-8555 https://orcid.org/0000-0003-2546-0030 https://orcid.org/0000-0003-2651-5179 https://orcid.org/0000-0002-3159-103X https://orcid.org/0000-0002-8334-0267

\section{REFERENCES}

1. Singh A, Mann HS, Thukral CL, Singh NR. Diagnostic accuracy of MRCP as compared to ultrasound/CT in patients with obstructive jaundice. J Clin Diagn Res 2014;8:103-107.

2. Polistina FA, Frego M, Bisello M, Manzi E, Vardanega A, Perin B. Accuracy of magnetic resonance cholangiography compared to operative endoscopy in detecting biliary stones, a single center experience and review of literature. World J Radiol 2015;7:70-78.

3. Morris S, Gurusamy KS, Sheringham J, Davidson BR. Cost-effectiveness analysis of endoscopic ultrasound versus magnetic resonance cholangiopancreatography in patients with suspected common bile duct stones. PLoS One 2015;10:e0121699.

4. Giljaca V, Gurusamy KS, Takwoingi Y, et al. Endoscopic ultrasound versus magnetic resonance cholangiopancreatography for common bile duct stones. Cochrane Database Syst Rev 2015;CD011549.

5. Kondo S, Isayama $\mathrm{H}$, Akahane $\mathrm{M}$, et al. Detection of common bile duct stones: comparison between endoscopic ultrasonography, magnetic resonance cholangiography, and helical-computed-tomographic cholangiography. Eur J Radiol 2005;54:271-275.

6. Sotoudehmanesh R, Nejati N, Farsinejad M, Kolahdoozan S. Efficacy of endoscopic ultrasonography in evaluation of undetermined etiology of common bile duct dilatation on abdominal ultrasonography. Middle East J Dig Dis 2016;8:267-272.

7. Visrodia KH, Tabibian JH, Baron TH. Endoscopic management of benign biliary strictures. World J Gastrointest Endosc 2015;7:1003-1013.

8. Bruno M, Brizzi RF, Mezzabotta L, et al. Unexplained common bile duct dilatation with normal serum liver enzymes: diagnostic yield of endoscopic ultrasound and follow-up of this condition. J Clin Gastroenterol 2014;48:e67-e70.

9. Strongin A, Singh H, Eloubeidi MA, Siddiqui AA. Role of endoscopic ultrasonography in the evaluation of extrahepatic cholangiocarcinoma. Endosc Ultrasound 2013;2:71-76.

10. Zepeda-Gómez S, Baron TH. Benign biliary strictures: current endoscopic management. Nat Rev Gastroenterol Hepatol 2011;8:573-581.

11. Rösch T, Meining A, Frühmorgen S, et al. A prospective comparison of the diagnostic accuracy of ERCP, MRCP, CT, and EUS in biliary strictures. Gastrointest Endosc 2002;55:870-876.

12. Rana SS, Bhasin DK, Sharma V, Rao C, Gupta R, Singh K. Role of endoscopic ultrasound in evaluation of unexplained common bile duct dilatation on magnetic resonance cholangiopancreatography. Ann Gastroenterol 2013;26:66-70.

13. Kim JE, Lee JK, Lee KT, et al. The clinical significance of common bileduct dilatation in patients without biliary symptoms or causative lesions 
on ultrasonography. Endoscopy 2001;33:495-500.

14. Oppong KW, Mitra V, Scott J, et al. Endoscopic ultrasound in patients with normal liver blood tests and unexplained dilatation of common bile duct and or pancreatic duct. Scand J Gastroenterol 2014;49:473-480.

15. Malik S, Kaushik N, Khalid A, et al. EUS yield in evaluating biliary dilatation in patients with normal serum liver enzymes. Dig Dis Sci 2007;52:508-512.

16. Singh A, Gelrud A, Agarwal B. Biliary strictures: diagnostic considerations and approach. Gastroenterol Rep (Oxf) 2015;3:22-31.

17. Ding $\mathrm{H}$, Zhou $\mathrm{P}, \mathrm{Xu} \mathrm{M}$, et al. Combining endoscopic ultrasound and tumor markers improves the diagnostic yield on the etiology of common bile duct dilation secondary to periampullary pathologies. Ann Transl Med 2019;7:314

18. Fritscher-Ravens A, Broering DC, Knoefel WT, et al. EUS-guided fine-needle aspiration of suspected hilar cholangiocarcinoma in potentially operable patients with negative brush cytology. Am J Gastroenterol 2004;99:45-51.

19. Eloubeidi MA, Chen VK, Jhala NC, et al. Endoscopic ultrasound-guid- ed fine needle aspiration biopsy of suspected cholangiocarcinoma. Clin Gastroenterol Hepatol 2004;2:209-213.

20. Lee JH, Salem R, Aslanian H, Chacho M, Topazian M. Endoscopic ultrasound and fine-needle aspiration of unexplained bile duct strictures. Am J Gastroenterol 2004;99:1069-1073.

21. Hashmi MAK, Jun LZ. Role of endoscopic ultrasound (EUS) in uncertain diagnosis of distal common bile duct lesions: a case study. IOSR Journal of Dental and Medical Sciences (IOSR-JDMS) 2018;17:72-75.

22. Ledro-Cano D. Suspected choledocholithiasis: endoscopic ultrasound or magnetic resonance cholangio-pancreatography? A systematic review. Eur J Gastroenterol Hepatol 2007;19:1007-1011.

23. de Lédinghen V, Lecesne R, Raymond JM, et al. Diagnosis of choledocholithiasis: EUS or magnetic resonance cholangiography? A prospective controlled study. Gastrointest Endosc 1999;49:26-31.

24. Scheiman JM, Carlos RC, Barnett JL, et al. Can endoscopic ultrasound or magnetic resonance cholangiopancreatography replace ERCP in patients with suspected biliary disease? A prospective trial and cost analysis. Am J Gastroenterol 2001;96:2900-2904. 